\title{
Estilos de aprendizaje en estudiantes universitarios: recursos informáticos como estrategia para su evaluación
}

\author{
Marcio Alexander Castillo Díaz \\ Jorge Luis Mendoza Aly \\ Vicerrectoría de Orientación y Asuntos Estudiantiles \\ Universidad Nacional Autónoma de Honduras
}

.........

\section{Resumen}

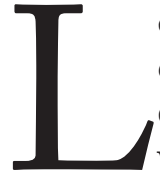

os cambios en la sociedad hacen que el proceso educativo esté en constante innovación y movimiento, provocando nuevas concepciones en el rol del docente y estudiante; las tendencias pedagógicas actuales priorizan centrar el proceso de enseñanza-aprendizaje directamente en el alumno, por tal razón resulta fundamental identificar formas en las cuales se adquiere, procesa y recupera la información, denominado por algunos teóricos de la psicología educativa como Estilos de Aprendizaje.

Los estilos de aprendizaje ayudan a los profesores a conocer más sobre los estudiantes a los que enseñan y a desarrollar efectivos modelos de desarrollo curricular, también contribuyen con los estudiantes a identificar la forma de cómo perciben, interaccionan y responden en ambientes de aprendizaje, así como desarrollar y aplicar estrategias que potencien su estilo de aprendizaje y permitan por consiguiente un mejor aprovechamiento académico.

El objetivo del presente estudio es describir los diferentes estilos de aprendizaje que presentan los estudiantes de primer ingreso en la UNAH y realizar una caracterización de los mismos en función del género y áreas del conocimiento a que pertenece cada uno. El instrumento utilizado fue el Modelo VARK en línea (Flemming \& Mills) el cual evalúa estilos de aprendizaje desde una modalidad sensorial basado en las categorías: Visual, Auditivo, Lecto-Escritura y Kinestésico. Para tal efecto, se aplicó la versión en línea de dicho instrumento, la cual se socializó a través de una herramienta de apoyo a la educación presencial diseñada y elaborada gracias a los cursos brindados por la Dirección de Innovación Educativa: los Blogs Educativos.
Palabras claves: Estilos de aprendizaje, VARK, Blogs Educativos, Estudiantes Universitarios, Áreas del Conocimiento

Abstract: Changes in society make the educational process to be constantly innovating and moving forward, causing new concepts in the role of teacher and student. Current educational trends prioritize focus the teaching-learning process directly to the student, for that reason it is essential to identify ways in which information is acquired, processed and retrieved, concept called by some theorists of educational psychology as "Learning Styles".

Learning styles help teachers to know more about the students they teach and to implement effective models of curriculum development; also, they contribute to the students to identify how they perceive, interact and respond to learning 
environments, furthermore to develop and implement strategies that enhance their learning style and thus enable a better academic performance.

The aim of this study is to describe the different learning styles that freshmen at UNAH have and make a characterization of them by gender and fields of knowledge. The instrument used was the "VARK Model" (Fleming \& Mills) which assesses learning styles from sensory modality based on the categories: $\mathrm{Vi}$ sual, Auditory, Read/Write and Kinesthetic. To this purpose, the online version of the instrument was applied, which it was conducted through a support tool for classroom education designed and developed through the courses offered by the Direction of Educational Innovation: "educational blogs".

Keywords: Learning styles, VARK, Educational blogs, University students, Fields of knowledge

\section{Introducción}

Los cambios que actualmente se viven en la sociedad hacen que el proceso educativo se encuentre en constante innovación y movimiento, provocando cambios en la concepción de los roles del docente y estudiante; las nuevas tendencias pedagógicas priorizan centrar el proceso de enseñanza-aprendizaje directamente en el estudiante, por tal razón resulta fundamental identificar las diversas formas en que ellos adquieren, procesan y recuperan la información.
La manera en que las personas adquirimos información puede ser categorizada de acuerdo a determinado estilo de aprendizaje. Actualmente existen muchos modelos teóricos que describen desde distintas perspectivas las "preferencias" o estilos de aprendizaje de cada individuo; muchos de estos modelos se agrupan principalmente en función de tres categorías en las que se focaliza su análisis: 1) Procesamiento de la información, en la que se incluyen diferencias en la cognición y percepción; 2) Patrones de personalidad, que involucra la influencia de variables motivacionales en el aprendizaje así como las características del ambiente y procesos de socialización, y 3) Modalidad sensorial, que incluye las reacciones con una base biológica a los estímulos del ambiente (Bernardes y Hanna, 2009).

Considerando que cada modelo teórico presenta fortalezas y limitantes, el conocimiento sobre los estilos de aprendizaje puede ayudar a los maestros a conocer más de los estudiantes a los que enseñan y a desarrollar -con base en ello- efectivos modelos de desarrollo curricular y de estrategias didácticas que propician la adquisición de aprendizajes significados por parte de los educandos; de igual manera el conocimiento sobre los estilos de aprendizaje es beneficioso para el propio estudiante, ya que al estar conscientes acerca de la mejor manera en que adquieren el conocimiento, pueden desarrollar técnicas y estrategias que potencien su aprendizaje.
El ingreso a la educación superior, para la mayoría de estudiantes, representa desafíos importantes en su adaptación, por el nivel de exigencia que las Universidades requieren. Ante ello, la Universidad $\mathrm{Na}-$ cional Autónoma de Honduras, definió como estrategia de abordaje el desarrollo de un Curso de Introducción a la Vida Universitaria, con la finalidad de contribuir a la formación integral del estudiante universitario, enfatizando en el desarrollo personal para la obtención de una mayor autonomía en el ámbito académico y social (CIVU, 2014).

Tal Curso se ha fortalecido en los últimos años mediante la incorporación de nuevas temáticas que permitan al estudiante no solamente conocer su institución sino también conocerse así mismo, principalmente en aspectos que conciernen a lo académico. Es por ello que como un componente innovador, se ha incluido la evaluación de los estilos de aprendizaje a través del modelo VARK, el cual clasifica por medio de un instrumento en línea (www.vark-learn. com), dichos estilos en función de modalidades sensoriales de los estudiantes.

\section{Objetivo General}

Caracterizar los estilos de aprendizaje que presentan los estudiantes de primer ingreso a la UNAH a través del instrumento en línea VARK; evaluando los resultados con base en dos variables demográficas: género y área del conocimiento. 


\section{Estilos de Aprendizaje}

El concepto de estilos de aprendizaje tiene sus antecedentes etimológicos a mediados del siglo XX en el campo de la psicología cognitivista, posterior a ello muchos estudios evidencian su grado de desarrollo o evolución, por ejemplo los que cita Valenzuela, Beltrona (2010) en su ponencia en el IV Congreso Mundial de Estilos de Aprendizaje: UNESCO (2005) , Rita Dunn, K. Kennet Dunn (1979), Gregory (1979), David Kolb (1976, 1984), Keefe (1982), y Alonso, Gallego \& Honey (2007), Valenzuela \& Hernández (2004), entre otros autores de reconocido prestigio. Aunque la bibliografía es muy amplia, existen diferencias o falta de consenso para definir un concepto para los Estilos de Aprendizaje; sin embargo, existen algunos que a lo largo de los años han sido mucho más acentuados y utilizados por quienes hacen uso del término.

Según Alonso et al (2007) 'Los Estilos de Aprendizaje son los rasgos cognitivos, afectivos y fisiológicos, que sirven como indicadores relativamente estables, de cómo los discentes perciben, interaccionan y responden a sus ambientes de aprendizaje”.

Kolb (1984) describe el concepto de estilos de aprendizaje como "algunas capacidades de aprender que se destacan por encima de otras, como resultado del aparato hereditario de las experiencias del medio ambiente actual".

De igual forma que existe una variedad de definiciones sobre el término, así encontramos también diferentes modelos para la evaluación de los estilos de aprendizaje, entre ellos:

Índice de Estilos Cognitivos de Allinson \& Hayes (CSI), Instrumentos de Estilos de Aprendizaje de Dunn \& Dunn model. Perfil de Estilos de Aprendizaje de $\mathrm{Vi}^{-}$ kkest lepe (LSP), Inventario de Estilos de Aprendizaje de Kolb (LSI), El cuestionario CHAEA de Estilos de Aprendizaje que es uno de los más utilizados en América Latina, y finalmente el instrumento VARK de Flemming \& Mills, modelo que será utilizado para el desarrollo de esta ponencia.

Es importante recordar que la definición de los estilos de aprendizaje de Alonso et. al. (2007) quien plantea rasgos cognitivos, afectivos y fisiológicos; sin embargo, el modelo empleado en la presente investigación se centra específicamente en características sensoriales, vistas como un componente de los rasgos fisiológicos que plantean los autores anteriormente citados.

\section{Estilos de Aprendizaje según Modelo VARK}

El Modelo VARK fue desarrollado por Fleming y Mills (1992) con la finalidad de ayudar a los estudiantes a entender y adaptar su preferencia individual de aprendizaje. Este modelo establece una caracterización de los estilos de aprendizaje con base en la modalidad sensorial con que se recibe y procesa la información por el aprendiz, dando como resultado cuatro
(4) diferentes estilos: visual, auditivo, lector/escritor y Kinestésico.

- Visual: esta modalidad incluye la preferencia por la información presentada en mapas, cuadros, gráficos, diagramas de flujo, organigramas, y otros recursos que utilizan los profesores para representar lo que podría haberse mostrado con palabras.

- Aural (Auditivo): Este modo de percibir describe una preferencia por la información "escuchada o hablada".

- Reading/Writing (Lecto-escritura): esta preferencia se refiere a la información mostrada en forma de palabras es decir que se centra en la información presentada en forma de texto -leer y escribir en cualquiera de sus formas.

- Kinestésico: esta modalidad se refiere a la "preferencia perceptual relacionada con el uso de la experiencia y la práctica (ya sea simulada o real)." Aunque esta experiencia puede evocar otras modalidades, la clave es que las personas que prefieren esta modalidad están conectadas con la realidad, "ya sea por medio de experiencias personales concretas, ejemplos, práctica o simulación" (Fleming \& Mills, 1992, pp. 140-141).

La base teórica del VARK se relaciona con los estudios realizados por Bruner (1967) y Piaget (1990) en los que describen cómo los humanos en general asimilan el conocimiento acerca de su entorno a través de tres modalidades
35 2015 
sensoriales: visual, auditivo y kinestésica; sin embargo, considerando los procesos sensoriales y perceptivos involucrados en la lecto-escritura, Flemings y Mills (1992) decidieron incluir esta última como una cuarta dimensión de su enfoque sobre los estilos de aprendizaje.

Fleming \& Mills (2001) afirman que aunque algunas personas pueden tener una fuerte preferencia por un único estilo de aprendizaje, muchos estudiantes pueden presentar afinidad por dos o más dimensiones sensoriales en los que procesan mejor la información. La combinación y preferencia por más de un estilo de aprendizaje es conocida como "bimodal" (en el caso de dos combinaciones), "trimodal"

36 (preferencia por tres combinaciones) y "multimodal" en aquellos sujetos con preferencia en las cuatro dimensiones que evalúa el VARK.

\section{Metodología}

\section{Participantes}

El total de participantes del estudio es 1,364 sujetos, todos ellos estudiantes que ingresaron a la Universidad Nacional Autónoma de Honduras (UNAH) durante $2013 \mathrm{y} / \mathrm{o}$ en los dos primeros períodos académicos de 2014, que se inscribieron en el Curso "Introducción a la Vida Universitaria" desarrollado por el Área de Orientación y Asesoría Académica de la Vicerrectoría de Orientación y Asuntos Estudiantiles (VOAE) y que contestaron el Cuestionario VARK en línea a través del
Blog Educativo del Curso: www.civuunah.wordpress.com

La muestra está compuesta por 844 mujeres (62\%) y 520 hombres (38\%), la media de edad correspondió a 19.45 con una desviación estándar de 4.04 años.

\section{Instrumento}

\section{El Cuestionario VARK} (VARK Questionnaire). Elaborado por Flemings y Mills (1992) y traducido al español por Sámano y Preciado (2007), es un instrumento diseñado para ser autoadministrado, así como de aplicación individual y/o grupal. Consta de 16 preguntas de opción múltiple que indagan la preferencia de las personas en capturar, procesar y entregar información basado en 4 diferentes modalidades sensoriales: visual, auditiva, lector/escritor y kinestésico.

La selección del cuestionario obedece a que es un instrumento conciso, accesible, económicamente asequible y fácil de contestar por parte de los estudiantes. El instrumento VARK fue calificado utilizando el algoritmo de calificación desarrollado por Flemings (1995).

\section{Procedimiento}

En el estudio participaron estudiantes universitarios de primer ingreso (aquellos con número de cuenta 2013 y 2014) matriculados en el Curso "Introducción a la Vida Universitaria" a participar en la investigación. Los estudiantes completaron el Cuestionario
VARK en el sitio web oficial del mismo (www.vark-learn. com), para lo cual se proporcionaron instrucciones específicas a través del blog educativo del Curso (www.civuunah. wordpress.com) sobre cómo contestar dicho instrumento y posteriormente registrar los resultados obtenidos a través de un formulario electrónico.

Sumado a lo anterior, para potenciar la utilidad del instrumento, se analizaron en el aula de clase los resultados obtenidos por los estudiantes y se les brindaron algunas estrategias y técnicas que ellos podrían utilizar para desarrollar un proceso de enseñanza-aprendizaje más provechoso considerando el estilo de aprendizaje predominante en cada uno.

Finalmente, con los resultados registrados a través del formulario se continuó con el procesamiento de los mismos a través del programa Microsoft Office Excel y su posterior análisis mediante el software estadístico IBM SPSS 22.

\section{Análisis de Resultados}

\section{Caracterización General}

Basado en los análisis estadísticos realizados, se encontró que el 39\% de los estudiantes presenta un estilo de aprendizaje unimodal (preferencia por una sola dimensión del VARK) frente a un $61 \%$ que muestra una preferencia multimodal (inclinación por más de un estilo de aprendizaje); de este último grupo de estudiantes el $20 \%$ evidencia un código bimodal, el 16\% trimo- 
dal y un $25 \%$ de los estudiantes se inclinan por una preferencia multimodal, es decir que muestran puntuaciones altas en las cuatro dimensiones del código VARK (Ver gráfico 1).

\section{-Estilos de aprendizaje en función del Género}

Como se observa en el gráfico 2 , en los datos analizados no aprecian diferencias significativas en función de la variable "género". De los tres indicadores con más de un estilo de aprendizaje, sólo en el bimodal es donde se aprecia un distanciamiento de 5 puntos porcentuales a favor de los hombres en comparación al estilo bimodal femenino.

En general, se identifica que los estudiantes del género masculino presentan mayor preferencia por al menos dos indicadores sensoriales que las del género femenino, reflejando mayor interés en códigos bimodales, trimodales y multimodales. En contraste, las mujeres reflejan con un $41 \%$ mayor interés por el aprendizaje mediante sólo una categoría, prevaleciendo con un 19\% el estilo Kinestésico seguido del Aural con $11.3 \%$.

-Estilos de Aprendizaje en función de Áreas del Conocimiento

Las 1,364 respuestas obtenidas corresponden proporcionalmente a cuatro áreas del conocimiento según la siguiente descripción: Ciencias Biológicas y de la Salud 396 (29\%), Ciencias Sociales 395 (29\%), Ciencias Economo-administrativas 284 (20.8\%),

Gráfico 1: Modalidades de estilos de aprendizaje en estudiantes de primer ingreso a la UNAH años 2013 y 2014

Modalidades de estilos de aprendizaje en estudiantes de

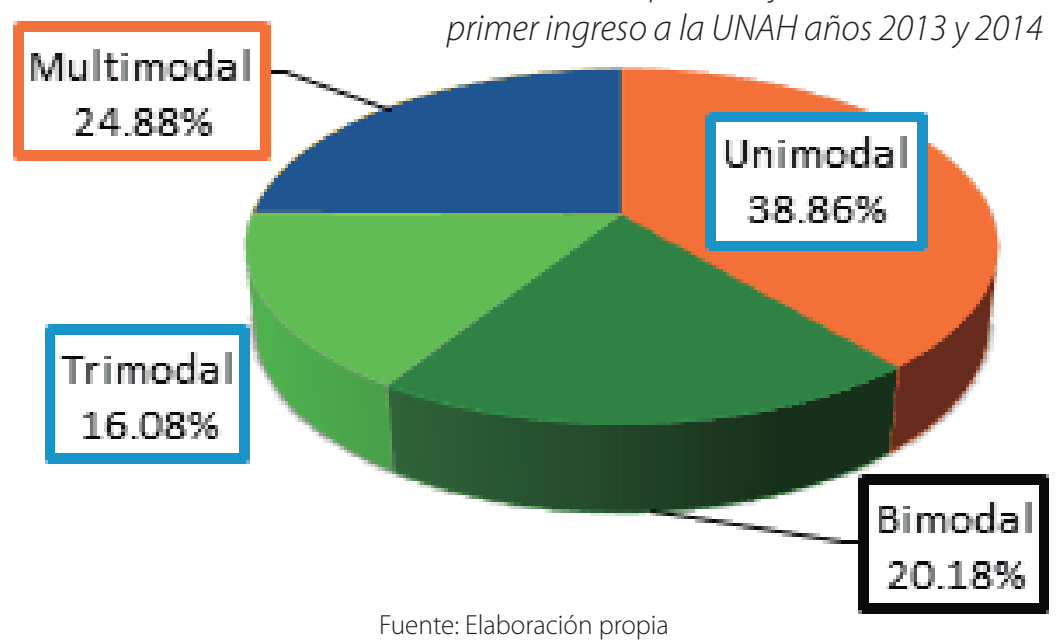

Gráfico 2: Modalidades de estilos de aprendizaje según el en estudiantes de primer ingreso a la UNAH años 2013 y 2014

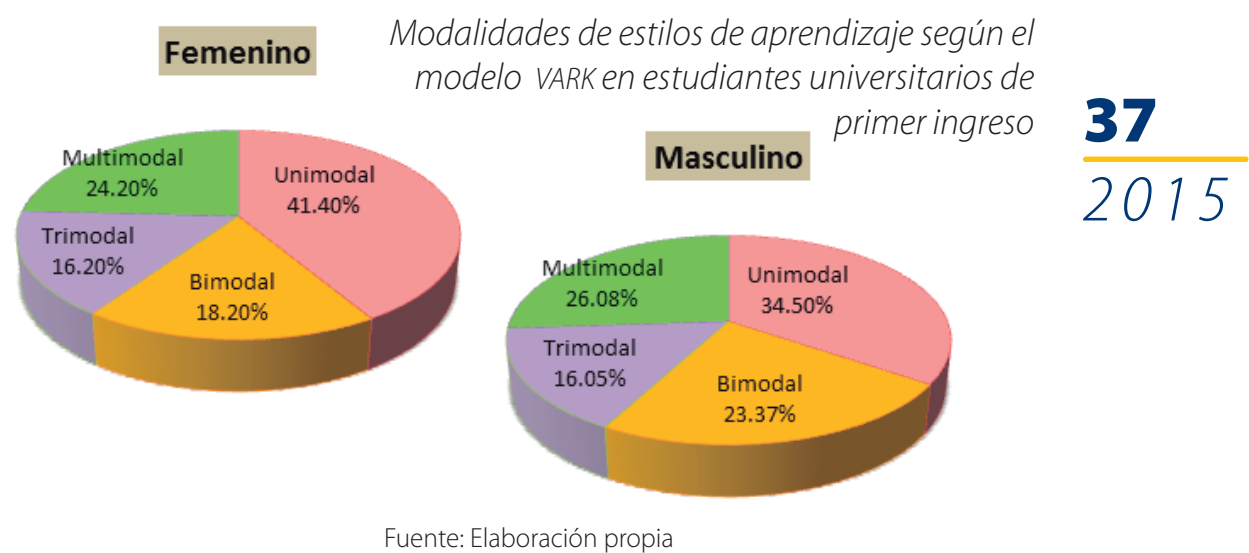

Ciencias Físico Matemáticas 282 (20.7\%) y Ciencias Agroforestales $7(0.5 \%)$.

La categoría Unimodal es la más representativa del estudio en cada una de las cuatro Áreas evaluadas (con porcentajes entre $34.4 \%$ en el Área Físico-matemática hasta $42 \%$ en el Área Econo-administrativa). Dentro de la Unimodalidad y tal como se observa en el gráfico 3 las cuatro áreas presentan los mayores porcentajes de preferencia en el estilo Kinestésico, con porcentajes que oscilan entre $14.1 \%$ y $18.2 \%$, haciendo una excepción en el Área Economo-administrativa donde los resultados son equiparables con el estilo $\mathrm{Au}-$ ral o Auditivo (14.1\%).

Los datos obtenidos durante el estudio indican que el estilo Visual cuenta con poca preferencia tanto en estudiantes unimodales, bimodales y trimodales. Los valores osci- 
lan entre 0.8\% (en el Área de las Ciencias Sociales) hasta 2.5\% (en el área de las Ciencias Econo-administrativas). Las diferencias porcentuales promedio entre las categorías Aural y Lectoescritura es bastante reducida, siendo apenas de un $2.25 \%$.

Basado en lo anterior, se puede concluir que los estudiantes con preferencia unimodal tienden a optar por tres de los cuatro estilos planteados por el Modelo, por tanto sus estrategias de estudio deben ser orientadas hacia el fomento de aplicaciones prácticas, el uso de canales auditivos como conferencias, debates, mesas de trabajo, ponencias así como la elaboración de ensayos, resúmenes o cualquier composición escrita elaborada por el estudiante.

\section{Conclusiones}

Los resultados evidencian que más de la mitad de los estudiantes que participaron en este estudio (61\%) tienen preferencia por más de un estilo de aprendizaje (bimodal, trimodal o multimodal); no existiendo diferencias significativas ni en función del sexo ni tampoco en base al área del conocimiento a la que pertenecen, reflejando esto una amplia diversidad respecto a la forma en que los estudiantes adquieren, procesan, retienen y recuperan la información.

Al utilizar el cuestionario VARK como instrumento para indagar los estilos de aprendizaje de los estudiantes de primer ingreso a la UNAH, se promueve la idea de que los educandos pueden aprender
Gráfico 3: Preferencia por estilo unimodal de aprendizaje según áreas del conocimiento

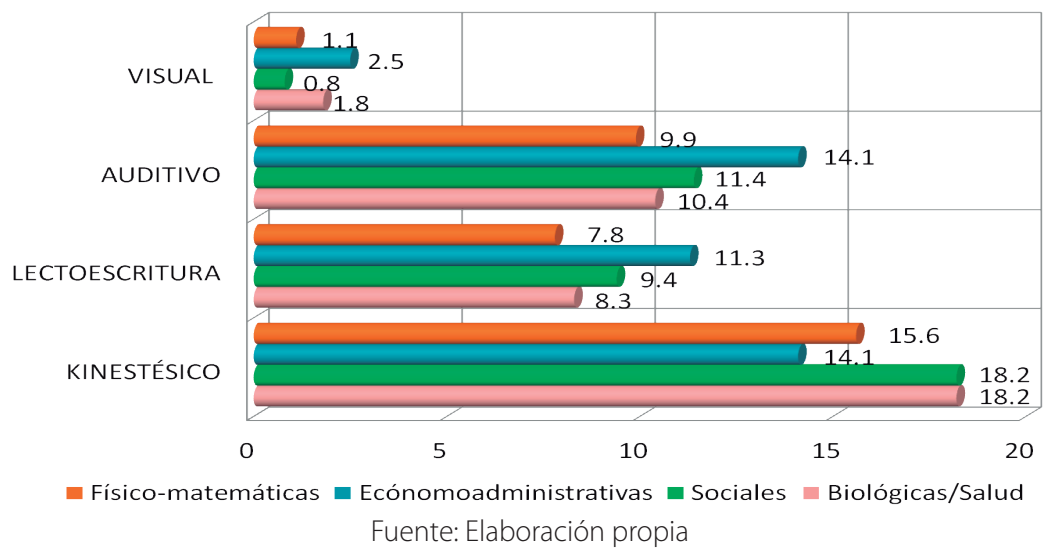

de distintas maneras; además mediante el enfoque investigación-acción que se ha utilizado se apoya a los estudiantes a que adquieran conocimiento de sus preferencias de aprendizaje y a que readecúen en función de ello sus técnicas de estudio de acuerdo a sus características individuales, contribuyendo así a lograr un mejor rendimiento académico.

Los resultados encontrados plantean un reto importante para los docentes universitarios, ya que al ser los facilitadores del proceso enseñanza-aprendizaje es fundamental que tomen en cuenta las preferencias y características individuales de sus estudiantes, ya que al tener conocimiento de tales diferencias en el aula de clase, pueden desarrollar así un amplio abanico de estrategias pedagógicas que propicien la adquisición de conocimientos significativos en los estudiantes.

Considerando la utilidad de las Tecnologías de la Información y Comunicación, el enfoque metodológico de recolección de resultados fue posible gracias a la utilización de un blog educativo, el cual es una de las herramientas educativas más valiosas que brinda la Web 2.0, esto plantea la posibilidad de realizar otros proyectos e intervenciones educativas que utilicen dicha herramienta tecnológica.

\section{Referencias}

Aguilera Pupo, E. y E. Ortiz Torres (2010) La caracterización de perfiles de estilos de aprendizaje en la Educación Superior, una visión integradora. Revista Estilos de Aprendizaje, No 5, Vol. 5, 26-41.

Alonso, C.M.; Gallego, D. J. y Honey, P. (2007) Estilos de Aprendizaje. Bilbao: Editorial Mensajero. 7a edición.

Área de Orientación y Asesoría Académica- UNAH (2014) Curso de Introducción a la Vida Universitaria. Ciudad Universitaria, Tegucigalpa, Honduras.

Beneitone, P. y Otros (2007) Reflexiones y perspectivas de la educación superior en América Latina: Informe final Proyecto Tuning-América Latina 2004-2007. Universidad de 
Deusto y Universidad de Groningen. http://tuning. unideusto.org/tuningeu/ (15 septiembre 2008)

Bernardes, E. \& Hanna, M. (2009) How do management students prefer to learn? Why should we care? International Journal for the Scholarship of Teaching and Learning. Giorgia, USA; 3 (1), pp. 1-12.

Bruner, J.S. (1967). Toward a theory of instruction. Cambridge: Harvard University press.

Dunn, R., Dunn, K. (1978). Teaching Students throught their Individual Learning Styles: A practical aproach. New Jersey: Prentice Hall.

Fleming, Neil. (1995) I'm different; not dumb: modes of presentation (VARK) in the tertiary classroom. Research and Development in Higher Education. Annual Conference of the Higher Education and Research Development Society of Australasia, Australia. 1995; vol. 18, pp. 308-313.

Fleming, Neil \& Mills, Colleen (1992). Not Another Inventory, Rather a Catalyst for Reflection. To improve the Academy, Vol. 11, Pág. 137.

Fleming, N. (2014) A guide to learning styles. Consultado en http://www.vark-learn. com/

Gregory (1979) "Modelos didácticos y estilos de Aprendizaje". Disponible en: www.usc.es/estaticos/co- nectate/conectate_programas/391/12502_1.htmconsultado-0302-09

Keefe, J. W, (1982). "Profiling and Utilizing Learning Style". Reston, Virginia: NASSP.

Kolb, D. (1984). Experiential Learning. Experience as the source of learning and development. Englewood Cliffs. New Jersey:Prentice Hall.

Ortiz, Edison; Sánchez, A. \& Lozano, A. (2013) REA $y$ estilos de aprendizaje según $V A R K$ en el aprendizaje de las matemáticas. Revista Internacional Magisterio: Educación y Pedagogía. 2013; 64, pp. 91-93.

Piaget, J. (1990). The child's conception of the world. New York: Littlefield Adams.

Recibido: 19/06/2015 